\title{
Pressure enhancement associated with meridional flow in high-speed solar wind: possible evidence for an interplanetary magnetic flux rope
}

\author{
C.-Y. Tu ${ }^{1,2}$, E. Marsch ${ }^{1}$, K. Ivory ${ }^{1}$, R. Schwenn ${ }^{1}$ \\ ${ }_{1}^{1}$ Max-Planck-Institut für Aeronomie, Postfach 20, D-37189 Katlenburg-Lindau, Germany \\ 2 Department of Geophysics, Peking University, Beijing 100871, China
}

Received: 24 January 1996 / Revised: 8 July 1996 / Accepted: 22 July 1996z

\begin{abstract}
A sizable total-pressure (magnetic pressure plus kinetic pressure) enhancement was found within the high-speed wind stream observed by Helios 2 in 1976 near $0.3 \mathrm{AU}$. The proton density and temperature and the magnetic magnitude simultaneously increased for about $6 \mathrm{~h}$. This pressure rise was associated with a comparatively large southward flow velocity component (with $V_{z} \approx-100 \mathrm{~km} \cdot \mathrm{s}^{-1}$ ) and magnetic-field rotation. The pressure enhancement was associated with unusual features in the electron distribution function. It shows a wide angular distribution of electron counting rates in the low-energy $(57.8 \mathrm{eV})$ channel, while previous to the enhancement it exhibits a wide angular distribution of electron count rate in the high-energy $(112,221$ and $309 \mathrm{eV}$ ) channels, perhaps indicating the mirroring of electrons in the converging field lines of the background magnetic field. These fluid and kinetic phenomena may be explained as resulting from an interplanetary magnetic flux rope which is not fully convected by the flow but moves against the background wind towards the Sun.
\end{abstract}

\section{Introduction}

In this short note we report the first (to our knowledge) observation of a magnetic flux rope in the bulk of an interplanetary high-speed stream.The data period under discussion showed all the typical characteristics of fast solar wind, such as lasting large-amplitude Alfvénic fluctuations and a low level of compressive fluctuations with weakly variable pressure. Yet, within such a typical fast stream a sizable total-pressure (magnetic pressure plus kinetic pressure) enhancement suddenly occurred and this was clearly evident in the temperature, density and magnetic magnitude, which all simultaneously increased for about $6 \mathrm{~h}$. This pressure rise was associated with a comparatively large southward deflection of the flow velocity (i.e. with a large out-ofecliptic component, $V_{z} \approx-100 \mathrm{~km} \cdot \mathrm{s}^{-1}$ ) and a distinct magnetic-field rotation. The pressure enhancement was neither associated with interactions between high-speed and low-speed wind nor related to a shock wave. These observations could not be explained as being due to a solar mass ejection (SME) with its typical signatures (for a review see, e.g., Gosling, 1990), but represent a new phenomenon.

There is convincing evidence that we are dealing here with an isolated flux rope of coronal-hole origin pushed by the fast wind to near convection, yet which slightly slips through the flow, and thus by lagging behind forms an obstacle for the wind by which it is deflected southwards. The flux rope is dynamically maintained by the wind pushing on it, such that the overall stress balance (or balance between differential ram pressure and total pressure) ensures stability of the rope, which otherwise would be expected to dissolve by a relaxation of the pressure excess through radiation of magnetoacoustic waves. The data discussed here have been analysed in Marsch and Tu (1993); they were looking for thermal- and magnetic-pressure correlations and identified this time as a period generally with anticorrelation at hourly scales, but with a sudden increase in the correlation coefficient between $p_{B}$ and $p_{k}$ to positive values larger than 0.5 during the present event, showing up clearly in their Fig. 9, last panel, after day 110 of 1976, and in the corresponding survey plot of the relative pressure amplitude in Fig. 2b, lowest panels, of Tu and Marsch (1994). Yet, it will later become clear that this strong pressure pulse is not related to a propagating magnetosonic wave but to the pile-up of the pressure of magnetosonic fluctuations caused by the plasma flow.

\section{Observations}

Figure 1 shows the time-sequence of several plasma parameters measured by Helios 2 in a two-day period beginning with hour 10 on day 110 in 1976 . The data are averaged over $405 \mathrm{~s}$ (corresponding to ten measurement cycles). The radial component of the velocity is shown in the first panel to indicate the overall stream structure. The wind speed decreases from about 750 to $650 \mathrm{~km} \cdot \mathrm{s}^{-1}$ 


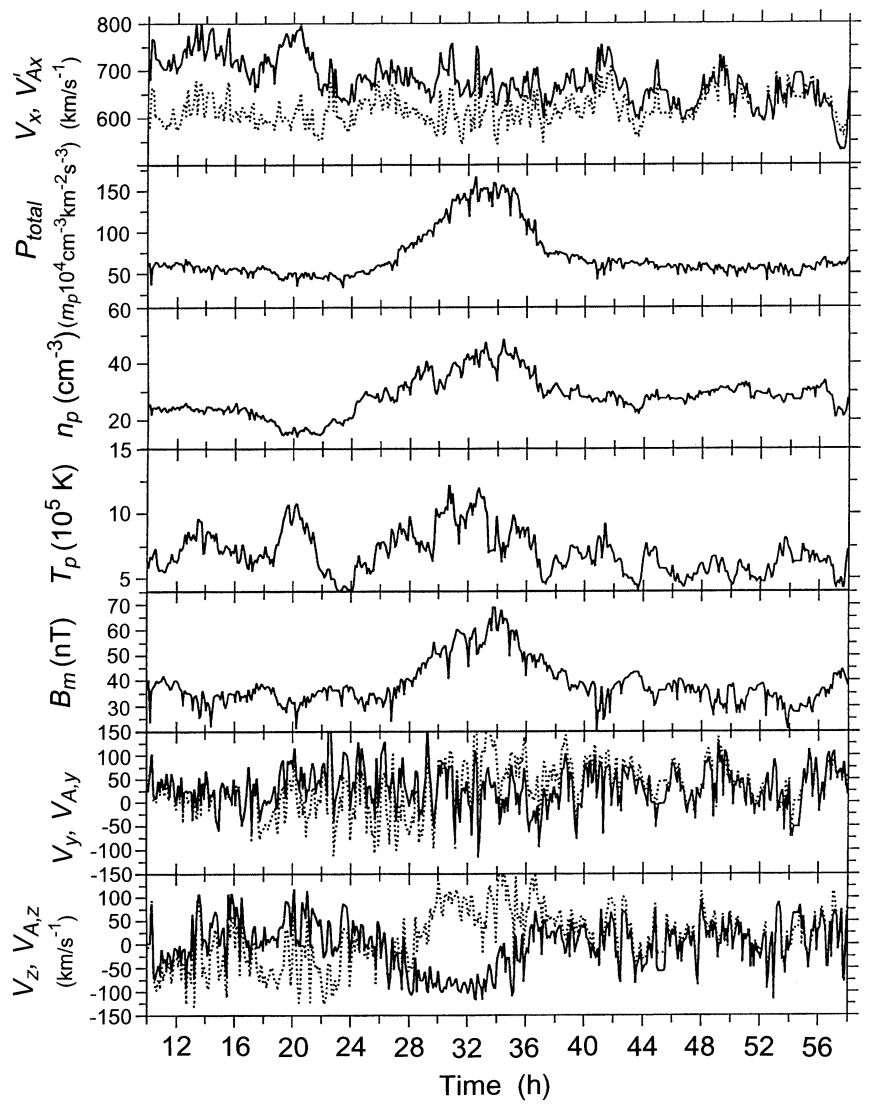

Fig. 1. Time-sequence of solar-wind parameters as observed by Helios 2 from day 110, hour 10, to day 112, hour 10, in 1976. From the top panel to the bottom panel we have: the radial components of the flow velocity (solid line) and Alfvén velocity (plus $750 \mathrm{~km} \cdot \mathrm{s}^{-1}, V_{A}^{\prime}$ ) indicated by a dotted line; the total pressure (electron temperature is assumed to be equal to the proton temperature) in units of $m_{p}$ $10^{4} \mathrm{~cm}^{-3} \mathrm{~km}^{2} \cdot \mathrm{s}^{-2}$; the proton number density; the proton temperature; the magnetic-field magnitude; the $y$ and $z$ (bottom panel) components of the solar-wind bulk velocity and of the Alfven velocity. The data were averaged over $405 \mathrm{~s}$, corresponding to ten plasma instrument measurement cycles. Note the pronounced pressure pulse amd magnetic-field enhancement characterizing the magnetic flux tube

in this period, which corresponds to the trailing edge of the high-speed wind observed by Helios 2 near $0.3 \mathrm{AU}$ in its first perihelion passage. No stream compression effects should be expected in the bulk of this stream far away from stream interaction regions or magnetic sector boundaries.

The total pressure (whereby we assume $T_{e}=T_{p}$ ) is shown in the second panel of Fig. 1. A clear totalpressure enhancement is visible between hours 28 and 37 , with the maximum pressure being three times larger than its ambient value. A special trait of this pressure pulse is that its trailing edge (hours 34 to 37 ) is somewhat steeper than its leading edge ( hours 26 to 32 ). From the third, fourth and fifth panels, one can see that the pressure enhancement is associated with enhancements in proton density, proton temperature and the magnetic magnitude, which rises by a factor of two during this event. The maximum proton tempera- ture is larger than one million degrees. The positive correlations between these three parameters indicate that strong (fast-mode wave type) compression takes place in this event.

The last two panels of Fig. 1 show the time-sequences of the $y$ and $z$ components (in solar ecliptic coordinates) of both flow velocity and Alfvén velocity $\left(V_{A}\right)$, plotted as the solid line and the dotted line, respectively. Normally, these two lines would appear close together (see e.g. the hours 40 to 58 and hours 10 to 12), indicating the typical correlation for outwards-propagating Alfvén waves. The dotted line in the first panel shows $V_{A, x}+750 \mathrm{~km} \cdot \mathrm{s}^{-1}$, where the constant speed is added to compensate the larger radial flow velocity component of about $650 \mathrm{~km} \cdot \mathrm{s}^{-1}$, and to make the average $V_{A, x}$ of about $-100 \mathrm{~km} \cdot \mathrm{s}^{-1}$ comparable with the radial velocity fluctuations. We can see good correlations in all the three components. However, during the pressure enhancement and in the ten hours preceding that event the correlations at the hourly scale are not good, especially in the $z$ component in the bottom panel showing large discrepancies.

In order to study the details of the high-pressure event, we show the kinetic pressure (solid line) and magnetic pressure (dotted line) obtained from hightime-resolution data (averaged over 81 s, i.e. two measurement cycles) in the first panel of Fig. 2. There is no indication of a shock wave; but there are some jumps in both curves, though they are related with pressure-balanced structures. To see the directed smallscale flows more clearly, we erased the small-scale Alfvén waves by application of gliding averages on several plasma parameters with a time window of $1 \mathrm{~h}$ and a shift of $81 \mathrm{~s}$. The results are shown in the second to fifth panels. In the second panel we can see that the pressure pulse is associated with a large meridional flow. Between hours 29 and 33 the component $V_{z}$ is near $-80 \mathrm{~km} \cdot \mathrm{s}^{-1}$. At the same time, $B_{z}$ and $B_{y}$ are positive, while the field angles are such that $\theta \sim 30^{\circ}$ and $\phi \sim 30^{\circ}$. The plasma beta is shown in the sixth panel. It varies in the range from 0.5 to 1.4 . Low beta values correspond to pressure-balanced structures, visible by the marked anticorrelations of the two traces in the first panel of Fig. 2, with a larger magnetic than thermal pressure.

The last panel shows the Alfvén ratio $\left(r_{A}\right)$ by the solid line and the normalized cross-helicity $\left(\sigma_{C}\right)$ by a dotted line, calculated for 1-h time-periods continually shifted by $81 \mathrm{~s}$. One normally finds $r_{A} \geq 0.8$ and $\sigma_{c} \geq 0.8$ being typical for Alfvénic fluctuations. However, from hours 30 to 34 , we see that $r_{A}$ is significantly lower; it can be as low as 0.5. Here the amplitude of the magnetic fluctuations is much larger than that of the velocity fluctuations. This reduction of $r_{A}$ and of $\sigma_{C}$ is associated with a southwards-oriented magnetic field and plasma flow.

In the following we present detailed electron azimuth-angle spectra with colour coding in order to illustrate typical features associated with the pressure enhancement and magnetic-field increase, which is expected to act as a magnetic mirror on the interplanetary electrons. Figure 3 shows in the top panel a 


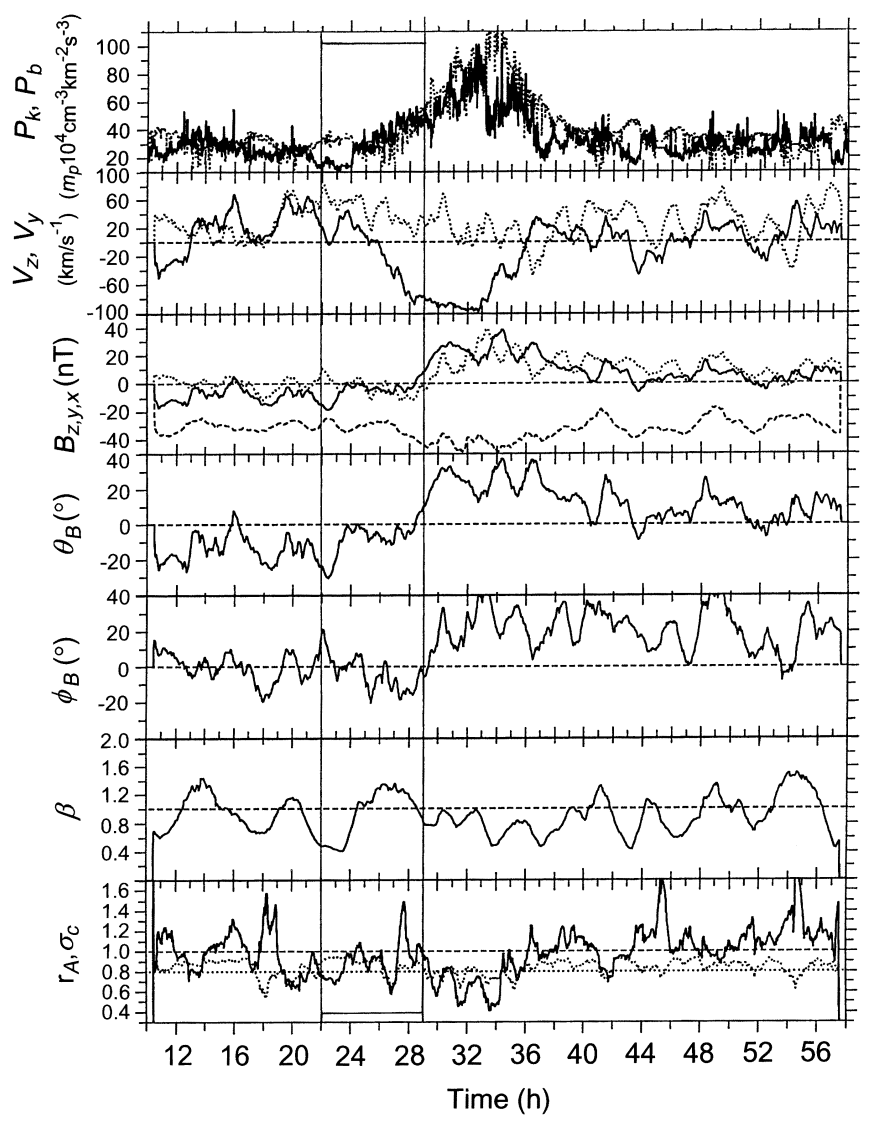

Fig. 2. Time-sequences of various parameters with different time resolutions for the same period as in Fig. 1. The first panel shows the high-time-resolution data of the total thermal pressure with the assumption of $T_{e}=T_{p}$ in units of $m_{p} 10^{4} \mathrm{~cm}^{-3} \mathrm{~km}^{2} \cdot \mathrm{s}^{-2}$ ) and the magnetic pressure indicated by the dotted curve. The original data were averaged over $81 \mathrm{~s}$. From the second to the fifth panel we show data obtained by averaging the original time-series over a 1-h-period window shifted continually by $81 \mathrm{~s}$. The parameters are: the $z$ (solid line) and $y$ (dotted line) component of the flow velocity; the $z$ (solid line), $y$ (dotted line) and the radial $x$ (dashed line) component of the magnetic-field vector; the elevation angle (in degrees) of the magneticfield vector with respect to the equatorial plane; the azimuthal angle of the magnetic-field vector; the plasma beta, i.e. the ratio between the thermal pressure and magnetic pressure; and finally in the last panel the Alfvén ratio (solid line), the ratio between the kinetic fluctuation energy and the magnetic fluctuation energy, and the normalized crosshelicity according to Roberts et al. (1987) given by the dotted line and calculated for a $1-\mathrm{h}$ period continually shifted by $81 \mathrm{~s}$

wide azimuthal-angle distribution of count-rates of electrons measured in the low-energy channel $(57.8 \mathrm{eV})$. The overall increase (from hours 28 to 38 ) in intensity, concurrently with the pressure enhancement, and the weak broadening of the distribution may have resulted from an adiabatic compression of electrons conserving their magnetic moment. The higher-energy channels (energies of 112 and $309 \mathrm{eV}$ ) show an even wider azimuth-angle distribution during hours 22 to 29 , and are indicated in Fig. 2 by the two vertical lines. This period just corresponds to the leading edge of the pressure increase, and also to the decrease in $V_{z}$. The electrons with a $90^{\circ}$ azimuth angle (if the azimuth angle of $\mathrm{B}$ is zero they would have a $90^{\circ}$ pitch angle) have zero parallel speed in the frame of the spacecraft. This isotropized distribution may result from a population of electrons with originally different parallel speeds, which may have been mirrored in the downward flow region where the background magnetic field lines are strongly converging, thus giving rise to the increase in the background magnetic field, which is shown for reference again in the bottom panel of Fig. 3 together with the elevation (out-of-ecliptic) angle of the magnetic field.

Figure 4 shows a hodogram of the magnetic field in principal-axis-analysis coordinates (PAA) for the time from hours 30 to 34 . This hodogram shows a turning around of the magnetic field during this period, although not an ideal complete rotation, displayed in the top panel in the maximum and intermediate variance components. The eigenvalues of the full variance matrix are 8.2, 17.3 and 89.0, increasing from minimum to maximum variance. The corresponding eigenvectors in Cartesian components read: $(1.94,0.30,-0.15),(-0.03$, $-0.35,-0.94)$ and $(0.34,-0.89,0.32)$. The related angles between the direction of minimum variance and the magnetic field and radial direction are $\Theta_{\mathbf{B}, \mathbf{M}}=135.1^{\circ}$ and $\Theta_{\mathbf{X}, \mathbf{M}}=19.7^{\circ}$. This variation of the field direction may be understood as being due to the flux tube geometry sketched in Fig. 5, which shows the flux rope, the possible mirror location, the stagnation point of the differential plasma flow, indicated by the thick arrow, and the localized southward deflection of the solar wind flow. Ideally, the second box of Fig. 4 should show a straight line parallel to the maximum variance axis, yet the data are not fully consistent with a pure rotation, because large-scale Alfvén waves may mix with the flux rope and disturb the rotational signature.

\section{Discussion}

The main pressure increase may be understood as being due to a strong compression resulting from the interaction between an interplanetary magnetic flux rope and the background solar-wind flow, as illustrated in Fig. 5. Thereby, the ram and thermal pressures of the wind are balanced by the magnetic stresses and pressure of the rope. The two side ends of the magnetic rope may be anchored in distant slow solar wind or still even be connected with the corona, or the rope may have just been disconnected from the corona, but its excess pressure and magnetic stress not yet been fully released, and thus the tube has not adjusted itself to the ambient fast wind. To support this interpretation we give a few quantitative considerations below.

We will assume that the negative value of $V_{z}$, as observed during hours 30 to 34 , is the result of a southward flow along a magnetic rope oriented in the direction of the average field during this period with angles $\theta=27.5^{\circ}$ and $\phi=113.2^{\circ}$. Such a deflection may result if the rope moves slower than the background flow with a speed difference of about $150 \mathrm{~km} \cdot \mathrm{s}^{-1}$, which would thus cause a pressure pile-up in front of the rope (during the time-span from hours 34 to 38, see previous figures). According to this model, the kinetic pressure of 

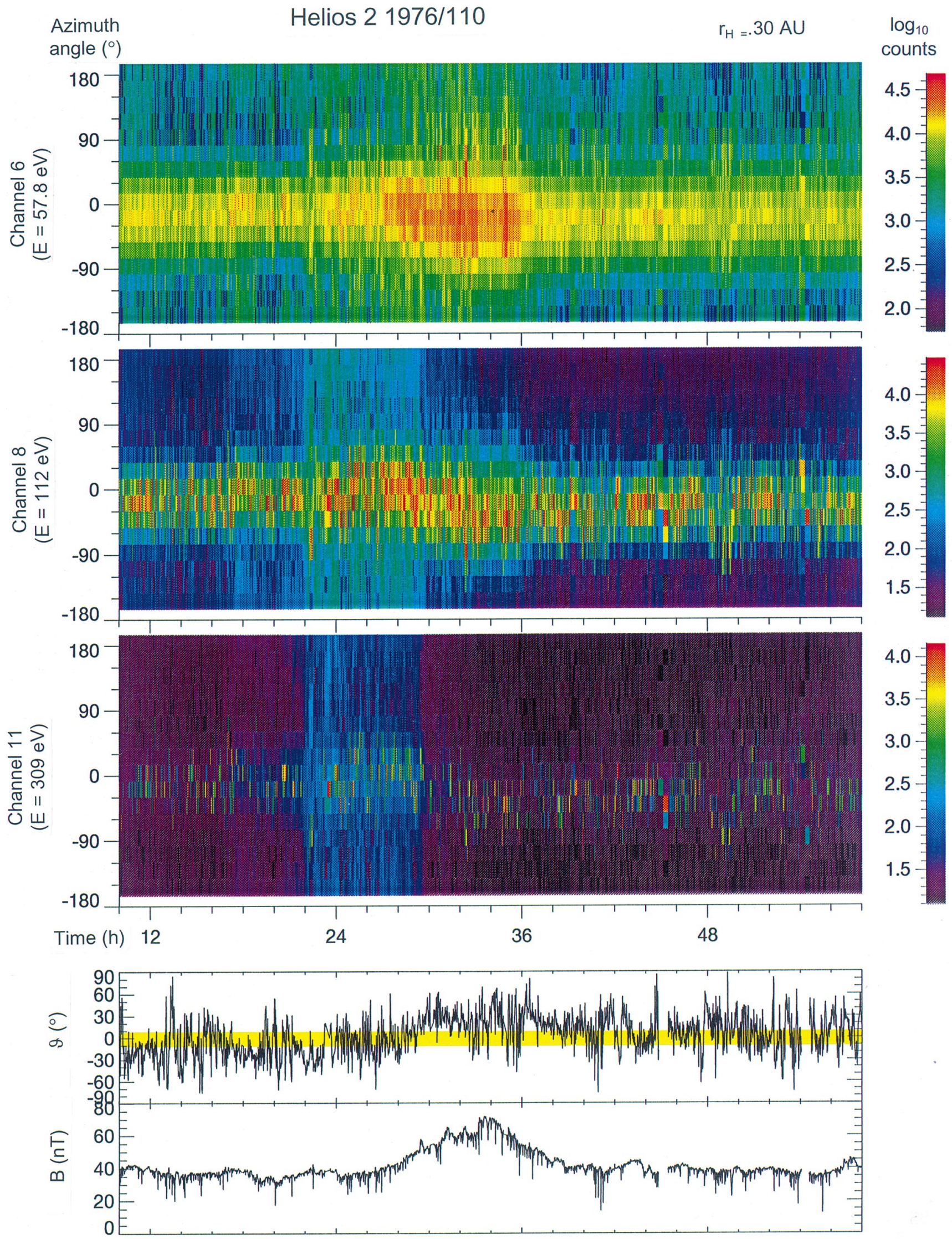

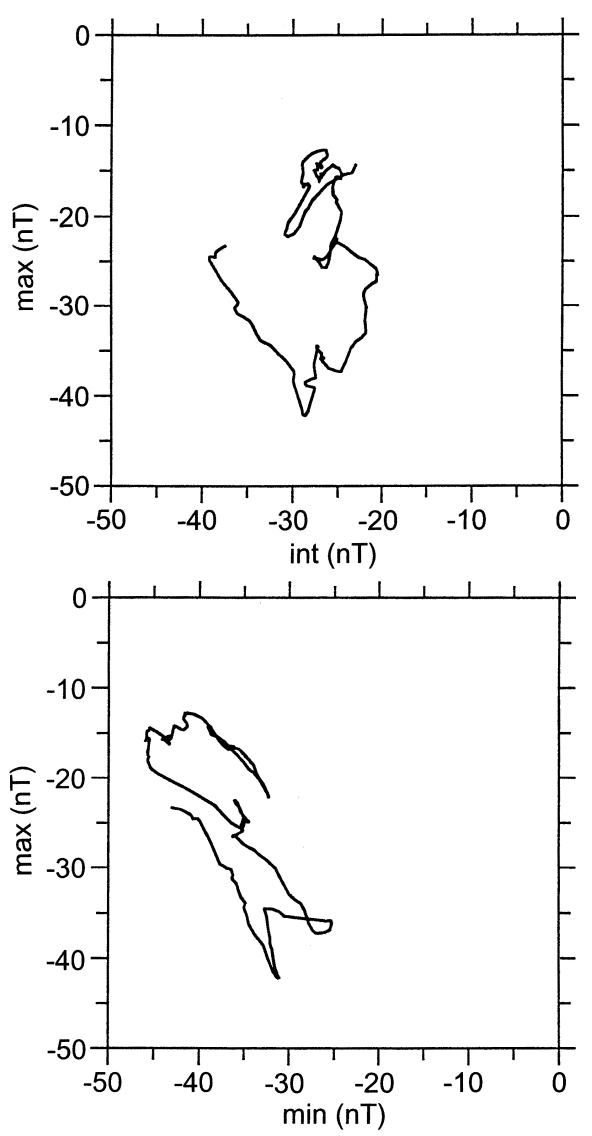

Fig. 4. Hodogram of the time variations of the magnetic-field components in principal-axis-analysis coordinates for the temporal variations during the hours 30 to 34 . Top: maximum and intermediate variance components; bottom: maximum and minimum variance components. Note the indications for a field rotation, interpreted as a trait of a magnetic flux rope

the background wind, as seen from a coordinate system moving with the magnetic flux rope, should just balance, in a dynamic equilibrium state, the total-pressure increase (from hours 34 to 38) of about 100 (in units of $m_{p} 10^{4} \mathrm{~cm}^{-3} \mathrm{~km}^{2} \cdot \mathrm{s}^{-2}$ ). If we use $\rho(\delta V)^{2}$ (with the mass density $\rho$ to evaluate the ram pressure at the stagnation point), we find that this kinetic pressure is 135 in the same units, which means it is of the same order as the total-pressure enhancement and thus sufficient to ensure dynamic force balance. A more detailed consideration of stress balance would require knowledge of the detailed local geometry of the event, which we do not have from the single-point measurements along the spacecraft

Fig. 3. Colour-coded azimuth-angle distributions of electron count rates obtained from Helios 2 in energy bands around 57.8, 112 and $309 \mathrm{eV}$ for an 38-h time-interval beginning at day 110 , hour 10, in 1976. The last two panels show the time variations of the elevation angle and the magnitude of the magnetic-field vector with a time resolution of $40.5 \mathrm{~s}$. Note the field enhancement and the associated features in the electron distributions, such as distinct broadenings in the distributions due to magnetic mirroring, particularly at high strahl energies in the third panel

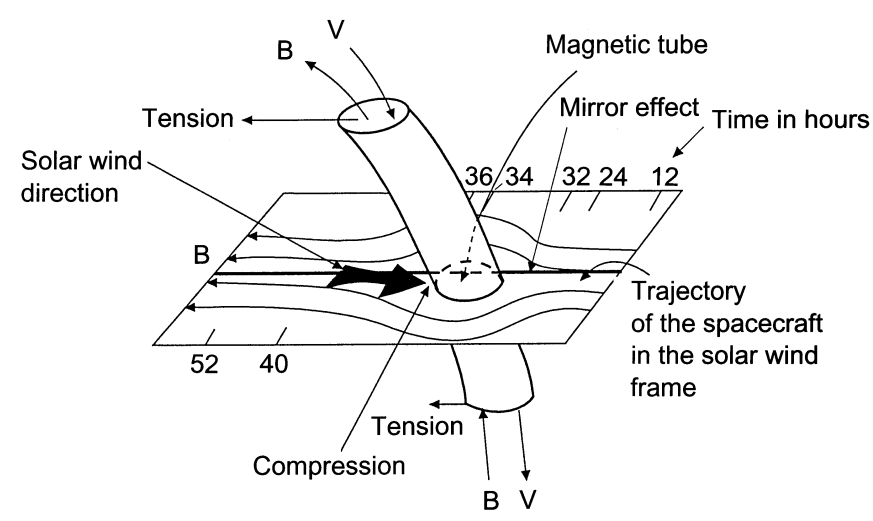

Fig. 5. Sketch of the assumed or inferred plasma flow (indicated by the thick large arrow) and magnetic-field geometry during the observed pressure enhancement, interpreted as evidence for an interplanetary magnetic flux rope that is partially convected by the flow, which by pushing on the tube maintains it in dynamic equilibrium. The Sun is to the left, which is the upstream direction

trajectory. Therefore, we shall be content with this simple analysis. Figure 5 presents a schematic of the geometry of the flux rope inferred from the data.

To summarize our findings: we have found, for the first time, evidence of an isolated interplanetary magnetic flux tube in fast solar wind. The plasma in the magnetic rope has a temperature higher than $10^{6} \mathrm{~K}$. The magnetic-field direction shows rotational features consistent with the flux-rope interpretation. The smallscale fluctuations in the plasma embedding the rope are mixed with largely magnetic variations, unlike the fluctuations in the ambient fast wind showing the signatures of Alfvén waves.

An investigation of the electron distribution functions in terms of count-rate spectra shows that the unusual azimuth-angle distributions of electrons at high energies may have been caused by the mirror effect of the converging magnetic field lines in the downstream region of the flow, where the background magnetic field lines are converging. This mirroring is typically observed at strong flux ropes associated with SMEs, which are known to form interplanetary magnetic clouds with strong field enhancements (see the review by Gosling, 1990), or most recently in Ulysses results on the possible occurrence of flux ropes in connection with interplanetary reconnection events (Moldwin et al., 1995), manifesting itself perhaps also by electron heat flux drop-outs.

Magnetic ropes (or clouds) have also been identified behind about one third of all interplanetary shock waves (Burlaga et al., 1981; Bothmer and Schwenn, 1994) caused by transient events in the solar atmosphere. A close association between erupting prominences on the Sun and magnetic clouds could be established. The magnetic structure of most erupting filaments is well reflected in the resulting magnetic-cloud topologies. Their main characteristic is a smooth rotation of the field vector nearly parallel to a plane over a time-interval of several hours. Furthermore, the plasma-beta values 
inside magnetic clouds are extremely low, since the field strength is high, while plasma density and temperature are very low. Also, the field is very quiet, without much fluctuation. The occurrence of bidirectional flows of suprathermal electrons indicates that either the field line they are moving on is rooted on the Sun at both ends, or that it is completely disconnected from the Sun (Gosling, 1990). The signatures of shock-associated magnetic clouds differ in many respects from those of the flux rope discussed here, and therefore we conclude that this rope has a different origin in the corona.

The origin of the flux tube embedded in a fast solarwind stream from a coronal hole remains unclear. It may have its cause in a small-scale flux tube (smaller than an eruptive prominence) on the Sun, which erupted or was dragged out by the fast stream and accelerated to partial convection with the wind.

Acknowledgements. The authors thank H. Rosenbauer and F. M. Neubauer for the use of the Helios plasma and magnetic field data. The Editor-in-chief thanks D. McComas for his help in evaluating this paper.

\section{References}

Bothmer, V., and R. Schwenn, Eruptive prominences as sources of magnetic clouds in the solar wind, Space Sci. Rev., 70, 215, 1994.

Burlaga, L. F., E. Sittler, F. Mariani, and R. Schwenn, Magnetic loop behind an interplanetary shock: Voyager, Helios, and IMP 8 observations, J. Geophys. Res., 86, 6673, 1981.

Gosling, J. T., Coronal mass ejections and magnetic flux ropes in interplanetary space, Geophys. Monograph, 58, 343, 1990.

Marsch, E., and C. Y. Tu, Correlations between the fluctuations of pressure, density, temperature, and magnetic field in the solar wind, Ann. Geophysicae, 11, 659, 1993.

Moldwin, M. B., J. L. Phillips, J. T. Gosling, E. E. Scime, D. J. McComas, S. J. Bame, A. Balogh, and R. J. Forsyth, Ulysses observation of a noncoronal mass ejection flux rope: Evidence of interplanetary magnetic reconnection, J. Geophys. Res., 100, 19903, 1995.

Roberts, D. A., L. W. Klein, M. L. Goldstein, and W. H. Matthaeus, The nature and evolution of magnetohydrodynamic fluctuations in the solar wind: Voyager observations, J. Geophys. Res., 92, 11021, 1987.

Tu, C. Y., and E. Marsch, On the nature of compressive fluctuations in the solar wind, J. Geophys. Res., 99, 21481, 1994. 